\title{
Modélisation de la houle réelle appliquée au spectre de Pierson-Moskowitz
}

\section{Christophe Chevalier}

Doctorant, LMFGC, Université du Havre.

\author{
Martin Sanchez \\ Maitre de conférence, LMG, Université de Nantes.
}

\section{Résumé :}

Des enregistrements de houle réelle sont reproduits par superposition d'un grand nombre d'ondes sinusoïdales déphasées aléatoirement les unes par rapport aux aurres et dont les amplitudes satisfont une forme adimensionnelle du spectre de houle de Pierson-Moskowitz. Les signaux ainsi modélisés sont analysés statistiquement et les résultats obtenus sont confrontés aux valeurs théoriques avec succès. Par la suite, la corrélation existant entre la hauteur et la période des lames est mise en évidence. Enfin, l'application d'un critère de déferlement en profondeur infinie montre l'absence de vagues déferlantes.

\section{Introduction}

L'action du vent sur la surface libre de l'eau provoque des ondulations aléatoires. Cette action du vent se produit sur une zone finie appelée fetch sur laquelle une partie de l'énergie du vent est transférée au fluide de façon aléatoire. Lorsque l'énergie maximum a été atteinte pour chaque bande de fréquence, la mer est dite formée ou complètement développée. Un enregistrement de ce type de houle présente un fort caractère aléatcire; la description de la houle réelle apparaît donc comme relevant du domaine du traitement du signal. L'objet de cette étude est de reproduire des enregistrements de houle réelle de mer formée et mettre en évidence des caractéristiques inaccessibles par des méthodes directes.

\section{Spectre de houle}

\subsection{Définition}

Avec l'apparition de moyens de calculs de plus en plus performants, il a été possible de procéder à l'analyse spectrale d'enregistrement de houle et ainsi 
d'obtenir une représentation de la densité d'énergie en fonction de la fréquence. Cette méthode présente l'avantage de mettre en évidence de façon directe les fréquences prédominantes de la houle considérée.

Il s'est alors développé de nombreux modèles mathématiques de spectres de houle parmi lesquelles celui présenté par Pierson et Moskowitz [6] est sans doute le plus connu.

\subsection{Spectre de houle de Pierson at Moskowitz}

Des travaux antérieurs portant sur l'application de la théorie des similitudes aux problèmes de détermination du spectre de houle et l'analyse faite par Moskowitz de nombreuses données spectrales pour des mers complètement formées ont conduit Pierson et Moskowitz [6] à proposer l'expression suivante de l'énergie de la houle $S$ en fonction de la pulsation $\omega$ :

$$
S(\omega) d \omega=\frac{\alpha g^{2}}{\omega^{5}} \exp \left[-\beta\left(\frac{\omega_{0}}{\omega}\right)^{4}\right] d \omega
$$

avec les conventions suivantes :

- $\omega_{0}=\mathrm{g} / \mathrm{U}$ est la pulsation de référence, où $U$ désigne la vitesse moyenne du vent sur le fetch ;

- $\alpha$ est une constante sans dimension égale à $8,1.10^{-3}$;

- $\beta$ est une constante sans dimension égale à 0,74 .

Le moment d'ordre zéro du spectre $\mathrm{m}_{0}$ représente l'énergie total de la houle par unité de longueur et s'écrit sous la forme suivante:

$$
\mathrm{m}_{0}=\int_{0}^{\infty} S(\omega) \mathrm{d} \omega=\frac{1}{4 \beta} \frac{\alpha \mathrm{g}^{2}}{\omega_{0}^{4}}
$$

Les autres moments sont définis par:

$$
m_{k}=\int_{0}^{\infty} \omega^{k} S(\omega) d \omega
$$

\section{Analvse statistique d'un enregistrement de houle}

\subsection{Passage par des points singuliers}

Avant le développement de l'analyse spectrale, les enregistrements de houle étaient traités par des méthodes statistiques. Cartwright et Longuet-Higgins [2], considérant la houle réelle comme la superposition d'un ensemble d'oscillations 
diverses se propageant dans la même direction, ont fourni des premiers résultats théoriques d'ordre statistique intéressants. Ainsi, ils ont mis en avant les relations existant entre les différents moments d'ordre 0,2 et 4 du spectre et les fréquences de passage par des points singuliers de l'enregistrement telles que la fréquence de passage par le niveau moyen $\mathrm{N}_{0}$, ou par un maximum local $\mathrm{N}_{\max }$, permettant ainsi une estimation aisée des moments par une simple étude d'ordre statistique.

Par ailleurs, Cartwright et Longuet-Higgins obtiennent une formulation de la distribution des cotes des maximums locaux en fonction de l'unique paramètre $\varepsilon$ suivant :

$$
\varepsilon=\sqrt{1-\frac{m_{2}^{2}}{m_{0} m_{4}}}=\sqrt{1-\left(\frac{N_{0}}{2 N_{\max }}\right)^{2}}
$$

Ce paramètre est un indicateur de la forme caractéristique du spectre de houle et on l'appelle largeur de spectre. Les valeurs proches de zéro correspondent à des spectres étroits et une distribution des maximums de type loi de Rayleigh alors que pour des valeurs proches de l'unité, le spectre est à large bande et la distribution des maximums correspondante suit une loi de Gauss.

\subsection{Etude des hauteurs de lames}

Dans le cas d'un spectre de houle infiniment étroit $(\varepsilon=0)$, la répartition des hauteurs de lames suit la loi de Rayleigh suivante (Bonnefille, [1]) :

$$
f(H)=\frac{H}{4 m_{0}} \exp \left(-\frac{H^{2}}{8 m_{0}}\right)
$$

Cette expression permet de calculer différentes hauteurs caractéristiques parmi lesquelles on peut retenir :

- la hauteur moyenne des lames $H_{m}$ égale à $H_{m}=\sqrt{2 \pi m_{0}}$;

- la hauteur significative $\mathrm{H}_{1 / 3}$ définie comme la moyenne du tiers supérieur des plus hautes lames de valeur $\mathrm{H}_{1 / 3} \approx 4 \sqrt{\mathrm{m}_{0}}$.

\section{Modélisation d'un enregistrement de houle}

\subsection{Paramètres de référence}

Dans le but de mener une étude qui soit le plus général possible, nous avons travaillé sur une formulation adimensionnelle du modèle ce qui suppose le choix des paramètres de référence en ce qui concerne les variables homogènes aux longueurs et celles de type temporel. 
Concemant les longueurs, notre choix s'est porté sur la grandeur $\sqrt{m_{0}}$ suivant en cela les travaux de Cartwright et Longuet-Higgins [2].

Les variables temporelles sont, elles, rapportées à la période de référence $T_{0}$ déffnie par le spectre de Pierson et Moskowitz de la façon suivante :

$$
\mathrm{T}_{0}=\frac{2 \pi}{\omega_{0}}=2 \pi \frac{\mathrm{U}}{\mathrm{g}}
$$

alors que pulsations $\omega$ sont rapportées à la pulsation de référence $\omega_{0}$.

$$
\omega^{*}=\frac{\omega}{\omega_{0}}
$$

\subsection{Formulation du modèle}

La formulation présentée ci-dessous a déjà été utilisée auparavant, notamment par Karlsson [5] pour une étude bi-dimensionnelle de la réfraction de la houle réelle. Chakrabarti [3] propose également un aperçu de différentes méthodes. La dénivellation réduite de la surface libre $z^{*}$ est obtenue en sommant un grand nombre $N$ de vagues de pulsation réduite $\omega^{*}$ comprise dans lintervalle $0<\omega^{*}<\omega_{\max }^{*}$ où $\omega_{\max }^{*}$ est la pulsation réduite de coupare haute du spectre ce qui conduit à l'expression suivante :

$$
z^{*}\left(t^{*}\right)=\frac{z(t)}{\sqrt{m_{0}}}=\sum_{k=1}^{N} c_{k}^{*} \cos \left(2 \pi \omega_{k}^{*} t^{*}+\phi_{k}\right)
$$

avec les conventions suivantes

- les déphasages $\phi_{k}$ sont distribués aléatoirement sur $[0,2 \pi]$;

- les composantes $c_{k}^{*}$ sont définies comme suit :

$$
c_{k}^{*}=\sqrt{2 S^{*}\left(\omega_{k}^{*}\right) \Delta \omega^{*}}
$$

- $\Delta \omega^{*}$ est le pas de division sur les pulsations réduites $\Delta \omega^{*}=\omega_{\max }^{*} / \mathrm{N}$.

Le spectre de houle utilisé est une forme adimensionnelle du spectre de PiersonMoskowitz qui peut s'écrire de la façon suivante [4] :

$$
S^{*}\left(\omega^{*}\right) \mathrm{d} \omega^{*}=\frac{S(\omega) \omega_{0}}{m_{0}} \mathrm{~d} \omega^{*}=4 \beta \frac{1}{\omega^{*^{5}}} \exp \left(-\frac{\beta}{\omega *^{4}}\right) \mathrm{d} \omega *
$$

et dont le moment d'ordre zéro réduit $\mathrm{m}_{0}^{*}$ est égale à l'unité (voir figure 1).

La nécessité d'effectuer une coupure à pulsation haute du spectre nous a conduit à prendre $\omega_{\max }^{*}=5$, pulsation pour laquelle plus de $99 \%$ de l'énergie est prise en compte. Ceci conduit à une largeur de spectre égale à $\varepsilon=0,73$. Par ailleurs, nous avons choisi de prendre $\mathrm{N}=2048$ composantes pour reproduire la houle réelle. 


\subsection{Remarques sur la méthode employée}

Une originalité de ce travail consiste à choisir des pulsations réduites $\omega^{*}$ réparties aléatoirement entre 0 et $\omega_{\max }^{*}$ au lieu d'effectuer une discrétisation régulière de ce même domaine (méthode employée par Karisson, [5] ) ce qui introduit un caractère plus aléatoire au signal généré (procédé également suggéré par Chakrabarti, [3]). Par ailleurs, au lieu de reproduire un unique signal de très longue durée et de l'analyser, nous avons préféré calculer plusieurs signaux indépendants en réinitialisant à chaque fois les paramètres aléatoires pour enfin tirer des conclusions sur l'ensemble des enregistrements disponibles.

\section{Comparaison théorie - modèle}

\section{5,1 Comparaison avec les travaux de Cartwright et Longuet-Higgins}

\section{5:1.1 Estimation des moments du spectre}

La première étape consiste à retrouver les fréquences de passages par des points singuliers de la surface libre tels que les maximums locaux $\left(\mathrm{N}_{\max }\right)$ ou par le niveau zéro $\left(\mathrm{N}_{0}\right)$ par analyse statistique des signaux modélisés, les résultats théoriques étant fournis par la connaissance des moments du spectre étudié. On note que les écarts entre la théorie et les enregistrements reproduits sont très faibles (de l'ordre de $1 \%$, voir tableau 1 ).

Par suite, l'estimation des différents moments du spectre de houle à partir des précédents résultats conduit à une estimation des moments du spectre avec une bonne précision (voir tableau 2). Ces premiers résultats donnent une première indication favorable sur la validité de notre démarche.

\begin{tabular}{|c|cc|c|}
\hline GRANDEUR & \multicolumn{2}{|c|}{ THEORE } & MODELE \\
Expression & Valeur & Ecart relatif \\
\hline$N_{0}$ & $N_{0}=\frac{1}{\pi} \sqrt{\frac{m_{2}}{m_{0}}}$ & 2,42 & $+0,04 \%$ \\
\hline$N_{\max }$ & $N_{\max }=\frac{1}{2 \pi} \sqrt{\frac{m_{4}}{m_{2}}}$ & 1,76 & $+0,40 \%$ \\
\hline$\varepsilon$ & $\varepsilon=\sqrt{1-\frac{m_{2}^{2}}{m_{0} m_{4}}}$ & 0,73 & $-0,41 \%$ \\
\hline
\end{tabular}

Tableau 1 : Fréguences de passage par des points singuliers de la dénivellation 


\begin{tabular}{|c|c|c|c|}
\hline \multirow{2}{*}{ GRANDEUR } & Valeur & \multicolumn{2}{|c|}{ Estimation } \\
théorique & Expression & Ecart relatif \\
\hline $\mathrm{m}_{0}^{*}$ & 1,0 & $\mathrm{~m}_{0}^{*}=\sigma^{2}\left\{\mathrm{z}^{*}\right\}$ & $-0,40 \%$ \\
\hline $\mathrm{m}_{2}^{*}$ & 57,8 & $\mathrm{~m}_{2}^{*}=\pi^{2} \mathrm{~m}_{0}^{*} \mathrm{~N}_{0}$ & $-0,17 \%$ \\
\hline $\mathrm{m}_{4}^{*}$ & 7107,7 & $\mathrm{~m}_{4}^{*}=4 \pi^{2} \mathrm{~m}_{2}^{*} \mathrm{~N}_{\max }^{2}$ & $-1,00 \%$ \\
\hline
\end{tabular}

Tablean 2 : Estimation des moments du spectre par analyse statistique

\subsubsection{Distribution des maximums locaux}

Un histogramme de répartition de la cote de chaque passage par un maximum de la surface libre a également été effectué afin de comparer celui-ci à la loi de distribution démontrée par Cartwright et Longuet-Higgins. On note que la distribution trouvée est en parfait accord avec la théorie (voir figure 2).

\subsection{Etude des hauteurs de lames}

\subsubsection{Définition de la hauteur de lame employée}

Un point à noter tout de suite est la définition utilisée par la suite pour hauteur de lame. Deux façons de procéder sont possibles. La première consiste à prendre pour hauteur de lame la différence de niveau existant entre une crễte et le creux précédent alors que la seconde définit la hauteur de lame comme étant la différence maximale crête à creux existant pour des dénivellations de la surface libre comprises entre deux passages successifs par le zéro par valeurs décroissantes. C'est cette dernière définition que nous avons utilisé dans le cadre de notre étude, la période associée étant alors égale à lintervalle de temps séparant deux passages successifs par le zéro par valeurs décroissantes de l'enregistrement.

Notons également que la définition employée revient à ne retenir qu'une seule lame par cycle ainsi défini ce qui représente le cas particulier d'un enregistrement de houle pour lequel $2 \mathrm{~N}_{\max }=\mathrm{N}_{0}$ et par conséquent $\varepsilon=0$ (cf. équation 3).

\subsubsection{Distribution des hauteurs de lames}

Dans ces conditions, la distribution des hauteurs de lames tendrait vers une loi de Rayleigh ce que confirme l'histogramme de répartition que nous avons obtenu par exploration systématique d'un très grand nombre de lames (voir figure 3 ). 
On note toutefois une soüs-estimation de la densité de probabilité pour les hauteurs de fortes amplitudes et une surestimation pour les hauteurs intermédiaires.

\section{$\underline{5.2 .3 \text { Hauteurs remarquables }}$}

Nous avons également pu évaluer quelques hauteurs remarquables et les comparer avec les résultats prédits par la théorie (d'après Bonnefille, [1] ) ce qui conduit aux résultats figurant dans le tableau $\mathrm{n}^{\circ} 2$ (théorie pour $\varepsilon=0$ ). Nous constatons là aussi une bonne concordance avec la théorie, les écarts étant de l'ordre de $7 \%$ dans le cas le plus défavorable et pouvant s'expliquer par la différence existant entre la distribution théorique et celle obtenue par modélisation.

\begin{tabular}{|l|c|c|}
\cline { 2 - 3 } & THEORIE & MODELE \\
\hline Hauteur moyenne $\mathrm{H}_{\mathrm{m}} / \sqrt{\mathrm{m}_{0}}$ & 2,51 & $+3,6 \%$ \\
\hline Hauteur significative $\mathrm{H}_{1 / 3} / \sqrt{\mathrm{m}_{0}}$ & 4,005 & $-5,2 \%$ \\
\hline Hauteur $\mathrm{H}_{1 / 10} / \sqrt{\mathrm{m}_{0}}$ & 5,08 & $-6,7 \%$ \\
\hline
\end{tabular}

Tablean 3 : Hauteurs caractéristiques

\section{Corrélation hauteur - période}

\subsection{Corrélogramme hauteur - période}

Par la suite, on présente un corrélogramme hauteur - période des lames pour une mer formée à partir des nombreuses données obtenues par modélisation. La forme de ce corrélogramme permet de constater l'existence d'une relation particulière entre la hauteur et la période (voir figure 4).

On note que les hauteurs de lame importantes sont associées à des périodes importantes alors que les hauteurs les plus faibles sont associées à des périodes courtes de sorte qu'il n'existe pas de lames de grandes hauteurs ayant des petites périodes.

\subsection{Application au déferlement en profondeur infinie}

La forme particulière semble indiquer que les lames parmi les plus susceptibles de déferier ne sont pas présentes dans le signal reproduit grâce à notre modèle. Ceci nous amène à considérer le problème du déferlement à l'aide du critère de Michell en profondeur infinie. Présenté sous forme adimensionnelle et reporté sur le graphe représentant les différentes valeurs possibles des couples $\left(\mathrm{H}^{*}, \mathrm{~T}^{*}\right)$, le critère indique 
l'absence de lames déferlantes dans le cas de la houle ainsi modélisée (voir figure 5). Par ailleurs, la relative concordance existant entre la courbe limite de déferlement et la partie inférieure du corrélogrammme laisse supposer qu'il existe une relation entre le déferlement, qui constitue une limite physique au développement de la mer, et la forme particulière du spectre d'énergie de Pierson et Moskowitz.

\section{Conclusions}

La présente étude a permis de mettre en avant plusieurs résultats intéressants concernant la houle réelle satisfaisant à un spectre de houle de Pierson-Moskowitz parmi lesquels on peut retenir :

- Pour la définition de hauteur de lame employée, la distribution des hauteurs tend vers une loi de Rayleigh et ce quelque soit la largeur du spectre;

- Nous avons pu mettre en évidence la corrélation existant entre la hauteur et la période de lame;

- Enfin, la reproduction des enregistrements de houle et l'application du critère de Michell en profondeur infinie montre l'absence de lames susceptibles de déferler.

\section{Références bibliographiques}

[1]- Bonnefille, R., 1992, Cours d'hydraulique maritime, Editions Masson, 208 p. [2]- Cartwright D.E. \& Longuet-Higgins M.S., 1956, The statistical distribution of the moxima of a random function, Proc. $R$. Soc., Ser. A ,230, p. 212-232.

[3]- Chakrabarti, S.K., 1987, Hydrodynamics of offshore structures, SpringerVerlag, $440 \mathrm{p}$.

[4]- Chevalier C., 1995, La houle réelle dans une mer formée, modélisation appliquée au spectre de Pierson-Moskowitz, Mémoire D.E.A., Université de Nantes.

[5]- Karlsson T., 1969, Refraction of a continuous ocean wave spectra, Proc. ASCE, 95 (WW4), p. 437-448.

[6]- Pierson W.J. \& Moskowitz I., 1964, A proposed spectral form for fully developed wind seas based on the similarity of S.A. Kitaigorodskii, Journal of Geophysical Research, 69, p. 5181-5190. 


\section{Figures}

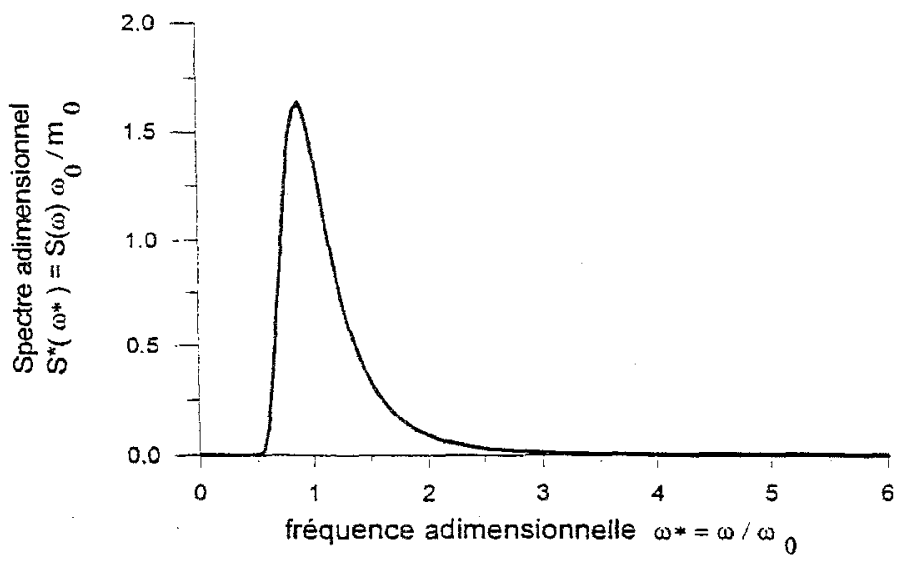

Figure 1: Forme adimensionnelle du spectre de Pierson-Moskowitz



Figure 2: Répartition des maximums locaux.



Figure 3: Répartition des hauteurs 


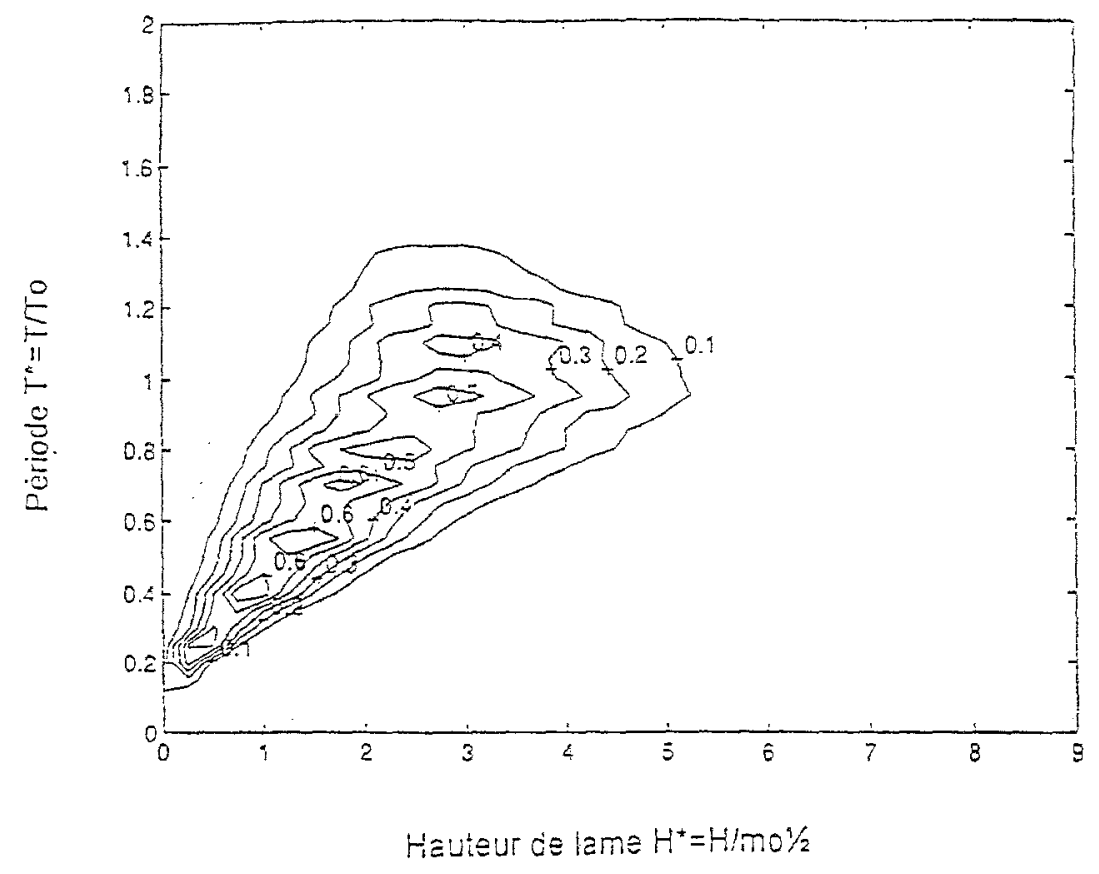

Figure 4: Corrélogramme hauteur-période



Figure 5: Application au déferlement en profondeur infinie 